Edith Cowan University

Research Online

Research outputs 2014 to 2021

$1-6-2021$

\title{
Predictors of endometrial carcinoma in patients with atypical endometrial hyperplasia at a tertiary gynaecological cancer centre in Western Australia
}

Vinita Angeline Rajadurai

Paola Chivers

Edith Cowan University

Chloe Ayres

Ganendra Raj Mohan

Colin John Reid Stewart

See next page for additional authors

Follow this and additional works at: https://ro.ecu.edu.au/ecuworkspost2013

Part of the Diseases Commons

10.1111/ajo.13300

This is an author's accepted manuscript of:

Rajadurai, V. A., Chivers, P., Ayres, C., Mohan, G. R., Stewart, C. J. R., Leung, Y. C., ... Cohen, P. A. (2021). Predictors of endometrial carcinoma in patients with atypical endometrial hyperplasia at a tertiary gynaecological cancer centre in Western Australia. Australian and New Zealand Journal of Obstetrics and Gynaecology, 61(2), 275-283.

https://doi.org/10.1111/ajo.13300

This Journal Article is posted at Research Online.

https://ro.ecu.edu.au/ecuworkspost2013/9703 


\section{Authors}

Vinita Angeline Rajadurai, Paola Chivers, Chloe Ayres, Ganendra Raj Mohan, Colin John Reid Stewart, Yee Chit Leung, King Man Wan, and Paul Andrew Cohen 
This is the peer reviewed version of the following article: Rajadurai, V. A., Chivers, P., Ayres, C., Mohan, G. R., Stewart, C. J. R., Leung, Y. C., ... Cohen, P. A. (2021). Predictors of endometrial carcinoma in patients with atypical endometrial hyperplasia at a tertiary gynaecological cancer centre in Western Australia. Australian and New Zealand Journal of Obstetrics and Gynaecology, 61(2), 275-283. https://doi.org/10.1111/ajo.13300, which has been published in final form at https://doi.org/10.1111/ajo.13300. This article may be used for non-commercial purposes in accordance with Wiley Terms and Conditions for Use of Self-Archived Versions. 


\section{Manuscript}

Predictors of endometrial carcinoma in patients with atypical endometrial hyperplasia at a tertiary gynaecological cancer center in Western Australia.

Key words: atypical endometrial hyperplasia, endometrial carcinoma, hysterectomy, predictor

Manuscript word count: 2498

Abstract word count: 240

Table count: 4

Figure count: 1 


\section{Abstract}

\section{Aim}

Our objective was to assess clinical and pathological factors associated with a final diagnosis of endometrial carcinoma in patients with atypical endometrial hyperplasia with a particular emphasis on the grading of atypia.

\section{Materials and methods}

A retrospective review over 5 years on patients $(n=97)$ who underwent hysterectomy for a diagnosis of atypical endometrial hyperplasia at a state-wide public tertiary gynaecologic oncology center. Clinical and pathological characteristics were obtained.

\section{Results}

The rate of concurrent endometrial carcinoma was $34 \%(n=33)$ with most being stage $1 \mathrm{~A}$ endometrioid. A significant group difference was reported for age at diagnosis $(t=-2.20 p=.031 d=0.43)$ with carcinoma patients on average older $\left(M_{a g e}=60.2\right.$ (8.9) years) than patients without carcinoma $\left(M_{a g e}=55.5\right.$ (12.3) years). No significant group differences were found for body mass index, endometrial thickness or time between diagnosis and treatment. Significantly higher rates of carcinoma were reported in patients with moderate atypical hyperplasia (27.6\%) and severe atypical hyperplasia (66.7\%), compared to mild atypical hyperplasia (7.1\%). Only severe atypical hyperplasia $(\mathrm{OR}=21.5,95 \% \mathrm{Cl} 2.8-163.1, \mathrm{p}=.003)$ and postmenopausal status $(\mathrm{OR}=13.2,95 \% \mathrm{Cl} 1.3-139.0, \mathrm{p}=.032)$ significantly increased the risk of carcinoma in a multivariate model.

\section{Conclusion}

Severe atypical hyperplasia and postmenopausal status were significant predictors of concurrent endometrial carcinoma in patients with atypical endometrial hyperplasia. The grading of atypical hyperplasia may be utilised by gynaecologic oncologists in the triage and referral process of managing these patients, however the grading system requires external validation in larger prospective studies. 


\section{Introduction}

Atypical endometrial hyperplasia (AEH), also referred to as endometrioid intraepithelial neoplasia, is considered a neoplastic process characterised histologically by an increased gland to stromal ratio within the endometrium accompanied by epithelial cytological atypia ${ }^{(1)}$. When focal, the morphological appearances of AEH are distinct from those of the adjacent non-atypical endometrium (2). Two classification systems for endometrial hyperplasia are in common use. The first, established by the World Health Organisation (WHO) in 1994, was based upon the sentinel retrospective analysis of 170 untreated endometrial hyperplasia patients by Kurman et al. Initially four types of hyperplasia were defined but later WHO revisions, most recently in 2020, have simplified endometrial hyperplasia into two main categories, atypical and non-atypical. The second main classification, the Endometrial Intraepithelial Neoplasia (EIN) system, was introduced in 2000 and based upon the integration of histological, molecular and objective morphometric criteria. Subsequent 'subjective' variations of this system have been proposed since these are more readily applicable in routine diagnostic practice (Baak and Mutter 2005, Owings and Quick 2014)

AEH coexists in $41-59 \%$ of patients with EAC $(4,5)$, and overall it is estimated that women with $\mathrm{AEH}$ have an $8 \%$ risk of developing EAC over 4 years ${ }^{(5-8)}$.

There can be a considerable range in the degree of architectural and cytological abnormality in cases of $\mathrm{AEH}$ and many studies have demonstrated inter-observer variation in diagnosis. Milder morphological alterations can overlap with reactive processes such as non-atypical hyperplasia or epithelial metaplasia whereas it may be difficult to distinguish more severe abnormalities from low-grade endometrioid adenocarcinoma. While there are no generally accepted or validated criteria for grading $\mathrm{AEH}$, in some centres pathologists therefore provide a subjective assessment of the degree of cytoarchitectural changes, generally categorised as mild, moderate or severe, since this may help to guide further management in individual patients $(7$, 9 , 10). For example, one retrospective study of 219 patients showed that the positive 
predictive value (PPV) of EAC following the diagnosis of mild-moderate AEH was $13 \%$ whereas severe $\mathrm{AEH}$ was associated with EAC in $50 \%$ cases ${ }^{(7)}$.

The incidence of $A E H$ is estimated to be three times that of EAC (11). AEH and EAC share risk factors including obesity and unopposed estrogenic effect that may be due to prolonged anovulation associated with perimenopause, polycystic ovarian syndrome, estrogen-secreting ovarian tumours, or exogenous estrogen use without a progestin for endometrial protection $(12,13)$. However, whether additional factors such as obesity and menopausal status modulate EAC risk in patients with AEH is unknown.

The recommended treatment for women with $\mathrm{AEH}$ is a hysterectomy due to the risk of concurrent or future malignancy (12). An alternative to patients who are not suitable for surgery, or who want to maintain fertility is hormonal therapy such as the Mirena ${ }^{\circledR}$ intrauterine system and/or oral progestin. The mainstay of treatment for EAC is surgical staging consisting of a total hysterectomy, bilateral salpingooophorectomy, pelvic and/ or para-aortic lymph node assessment (14). Nodal assessment is important in patients with accepted higher risk pathological features including high histological grade, deep myometrial invasion, presence of lymphovascular space invasion (LVSI), tumour size $>2 \mathrm{~cm}$, cervical stromal involvement and non-endometrioid tumour histology $(14,15)$. However, lymph node assessment is also important in women with presumed early stage, grade 1 EAC since up to $17 \%$ of patients may have occult nodal disease that would be otherwise undetected and untreated ${ }^{(16)}$.

Patients with EAC treated by gynaecologic oncologists tend to have superior outcomes to those treated by general gynaecologists ${ }^{(17)}$ likely due to comprehensive surgical staging, histological review by expert gynaecologic pathologists, and treatment recommendations made by a multidisciplinary team.

Currently, there are no reliable methods to determine which patients with AEH on endometrial biopsy are likely to have EAC on final histopathology, and therefore may 
benefit from gynaecologic oncology management. However, some studies have reported that type II diabetes, obesity, postmenopausal status and older age are associated with a final diagnosis of carcinoma ${ }^{(8,18-20)}$. Identification of such risk factors may assist in appropriate triage of patients suitable for management by gynaecologic oncologists.

The aim of the current study was to assess clinical and pathological factors associated with a final diagnosis of EAC in a cohort of patients with $A E H$, treated at a state-wide tertiary gynaecological cancer center. We hypothesized that the grade of AEH would be an important predictor of concurrent EAC.

\section{Materials and methods}

\section{Study design and Eligibility}

Ethical approval was granted (WA Health Governance Ethics Knowledge Outcomes reference no. 33346). Patients were eligible if they had a diagnosis of AEH on endometrial biopsy and subsequently underwent a hysterectomy. Exclusion criteria included patients who underwent hysterectomy without previously confirmed AEH and those with a confirmed or past history of EAC. Eligible patients between 2014 and 2019 were identified from an institutional surgical pathology database.

\section{Clinical information}

The general medical and gynaecological history of eligible patients was extracted from medical records and listed in Table $1-2$. The method of endometrial sampling was recorded [pipelle, dilation and curettage $(D \& C)$ ] or subsequent $D \& C$ if pipelle was undertaken initially. The treatment pattern was recorded including whether there was hormonal treatment between AEH diagnosis and hysterectomy, type of hormonal agent, time interval between initial diagnosis of $\mathrm{AEH}$ and the date of hysterectomy, and the operating team. 
Histopathology data included the grade of AEH in the initial biopsy (classified as mild, moderate or severe), and suspicion or inability to exclude malignancy. Additional data included whether AEH was confined to a polyp and the cytology of pelvic washings if performed. For patients diagnosed with EAC on subsequent hysterectomy, histological subtype, grade, depth of myometrial invasion, tumour size, stage, presence of lymphovascular space invasion, and, where applicable, lymph node status and frozen section findings were recorded.

\section{Histopathological grading classification}

As noted above, there are no validated criteria for grading $A E H$, but as in some other centers $(7,9)$ it has been local practice in Western Australia for pathologists to subjectively assess the degree of architectural and cytological atypia on a 3-point scale. All cases by definition demonstrate increased gland to stromal ratio in comparison with non-hyperplastic endometrium with in addition cytological atypia of the epithelium. Grading is predominately based upon the degree of glandular crowding with the epithelial component of mild, moderate and severe AEH accounting for approximately $50-65 \%, 66-80 \%$ and $>80 \%$ of the mucosal area, respectively (Figure 1). A panel of gynaecologic pathologists, as described previously $(21,22)$, reviews cases of AEH presented at the Western Australian Gynaecologic Cancer Service (WAGCS) to minimise inter-observer variation in diagnosis.

\section{Management of atypical endometrial hyperplasia}

WAGCS provides tertiary care for the state of Western Australia, which has a population of more than 2.5 million people. All cases of AEH at our centre and cases referred from peripheral sites are reviewed at a centralized, statewide multidisciplinary tumour board. Following tumour board and central pathology review, gynaecologic oncologists manage patients with severe AEH. Historically, we performed hysterectomies for patients with all grades of AEH, but since 2018 patients with mild and moderate $\mathrm{AEH}$ are managed by their referring general gynaecologist. We aim to see all patients with AEH within four weeks and triage as category 1 elective surgery (needing treatment within 30 days). 


\section{Statistical analysis}

Data was analysed using IBM Statistical Package for Social Sciences (SPSS) Statistics for Windows, version 26 (IBM Corp., Armonk, N.Y., USA), with an alpha of .05 considered statistically significant. Categorical variables were described using frequency and percent, with missing data noted. Continuous scale variables were described using mean, median and standard deviation. Group differences between carcinoma (present/absent) and each categorical variable were examined using Fisher's Exact and Chi-square tests with Cramer's $V$ effect sizes reported and interpreted as small 0.1 , medium 0.3 and large 0.5 (two categories); small 0.07 medium 0.21 and large 0.35 (three categories) and small 0.06 , medium 0.17 and large 0.29 (four or more categories). Group differences for categorical scale variables were examined using the independent t-test for BMI and age at diagnosis (with Hedges's $d$ adjusted Cohen's $d$ effect size), and the non-parametric MannWhitney $U$ Test (with Eta squared ( $n 2$ ) effect size) for endometrial thickness and days from diagnosis to treatment (based on the Shapiro-Wilk test for normality). Both Hedges's $d$ and $\eta 2$ were calculated and interpreted using Lenhard and Lenhard's (2016) online calculator and interpretation table ${ }^{(23)}$.

A binary logistic generalized linear model (GLM) was used to examine the odds ratio (OR) for carcinoma (absent set as the reference category) with the statistically significant variables from the group differences analysis, including commonly controlled for confounders (regardless of statistical finding e.g. age at diagnosis and BMI). The binary logistic GLM with logit link function included atypical hyperplasia, suspicion of malignancy, hypertension, menopause status (binary: post menopause and pre/peri menopause) as fixed factors, and age at diagnosis, BMI and endometrial thickness as fixed covariates. A pairwise Bonferroni adjustment for multiple comparisons was conducted for atypical hyperplasia and menopausal status.

\section{Results}


There were 97 patients included in this study. Baseline data, general medical / gynaecological history and patient treatments are shown in Tables 1-2 respectively. Thirty-three patients (34.0\%) had a final diagnosis of EAC and their clinicopathological characteristics are summarised in Table 3. Most EACs were endometrioid, presumed stage $1 \mathrm{~A}$ (nodal status was not known in $93.9 \%$ of the cases) with no myoinvasion. The final histopathology was AEH only ( $n=39,40.2 \%$ ), carcinoma $(n=33,34.0 \%)$ and no residual AEH or carcinoma $(n=25,25.8 \%)$.

A significant group difference was reported for age at diagnosis $(t=-2.20 p=.031$ $d=0.43$ ) with carcinoma patients on average older $\left(M_{a g e}=60.2\right.$ (8.9) years) than patients without carcinoma ( $\mathrm{M}_{\mathrm{age}}=55.5$ (12.3) years). No significant group differences were found for $\mathrm{BMI}(\mathrm{t}=-0.41 \mathrm{p}=.681 \mathrm{~d}=0.09)$, endometrial thickness (Whitney $U z=0.98 p=.326 \eta^{2}=0.01$ ) or time between diagnosis and treatment (Mann-Whitney $U z=-0.47 p=.642 \eta^{2}=0.00$ ). Chi Square group differences examined for each factor are summarised in Table 2. For menopausal status, premenopausal (13.0\%) and perimenopausal (16.7\%) had significantly lower rates of carcinoma compared to post-menopausal women (42.6\%). Only gynaecologic oncologists performed hysterectomies on all carcinoma patients $(n=33)$. Significantly higher rates of carcinoma were reported in patients with moderate $\mathrm{AEH}(27.6 \%)$ and severe AEH (66.7\%), compared to mild AEH (7.1\%). Where there was a suspicion of malignancy in the initial endometrial biopsy, $76.9 \%(n=10)$ of patients had a final diagnosis of carcinoma, where the opposite was true when there was no suspicion of malignancy $(72.6 \%$ no carcinoma). Although not statistically significant a higher proportion of hypertensive women $(42.1 \%)$ had carcinoma compared to those without hypertension (22.5\%) $\left(\chi^{2}=4.03 p=.052\right)$.

The sample for the binary logistic GLM was reduced to 71 patients due to missing data points across variables $(n=26[26.8 \%])$. This sample included 26 patients with carcinoma ( $78.8 \%$ of original 33 ) and 45 with no carcinoma (70.3\% of original 64$)$. Due to the small sample in perimenopausal women $(n=5)$, this group was combined with premenopausal women. The final model results are presented in Table 4. When all factors were combined, only severe $A E H(O R=21.5,95 \% \mathrm{Cl} 2.8-163.1, p=$ 
.003 ) and postmenopausal status (OR=13.2, 95\% $\mathrm{Cl} 1.3-139.0, \mathrm{p}=.032)$ significantly increased the risk of carcinoma. Pairwise comparisons indicated this increased risk was for severe AEH (compared to mild $p<.001$ and moderate $p=.001$ ) and for postmenopausal (compared to pre/peri menopausal $\mathrm{p}=.013$ ). Although not statistically significant, suspicion for malignancy and hypertension increased the risk of carcinoma by three- and two-fold respectively.

\section{Discussion}

The aim of the study was to assess clinical and pathological factors associated with a final diagnosis of EAC in patients with a preoperative diagnosis of $A E H$. In a multivariate model, severe $\mathrm{AEH}$ and postmenopausal status were significant predictors of EAC. Patients with moderate and severe AEH had significantly higher risks of EAC compared to those with mild AEH. These findings support a model of care where patients with moderate and severe AEH are managed by gynaecologic oncologists and patients with mild AEH are managed by gynaecologists.

Consistent with the findings of previous studies, 34\% of patients with $\mathrm{AEH}$ had a concurrent $\operatorname{EAC}(4,5,7)$. On univariate analysis patients with EAC were older, although age did not retain significance in a multivariate model. However, consistent with previous studies postmenopausal status was a significant predictor of concurrent $\operatorname{EAC}^{(8,18,24)}$.

$\mathrm{AEH}$ and EAC share risk factors and are strongly associated with obesity and diabetes due to higher circulating unopposed estrogen ${ }^{(12,13)}$. Previous studies have reported obesity $\left(\mathrm{BMI}>30 \mathrm{~kg} / \mathrm{m}^{2}\right)$ to be an independent risk factor for concurrent carcinoma $(8,19)$ so it is notable that BMI and diabetes mellitus were not associated with a final diagnosis of cancer in our study. This may be because our study was underpowered to detect such differences, as three-quarters of patients were obese and two-thirds of patients did not have diabetes, however effect sizes inferred no effect. Our findings are also not consistent with those of previous studies that have reported the 
risk of cancer to be lower in women for whom endometrial biopsies were obtained by $D \& C$ compared to those who had an office endometrial biopsy ${ }^{(25)}$. We did not observe a difference in EAC outcome according to the mode of endometrial sampling and this may be due to our relatively small sample and lack of statistical power, however the effect size inferred no effect.

Studies that have investigated hypertension as a risk factor for $\mathrm{AEH}$ and EAC have had inconsistent findings with some reporting an association and others failing to show significance $(8,19)$. We found that hypertension increased the risk of a concurrent EAC by two-fold although this was not significant in a multivariate model, with a small to medium effect size reported.

There is variation amongst practitioners in the preoperative workup and intraoperative management of patients with $\mathrm{AEH}$ both within Australia and internationally. Cost-effective, safe and accurate diagnostic modalities are needed to improve the preoperative and intraoperative identification of patients with concurrent EAC. Current literature on the accuracy of frozen section at the time of hysterectomy for AEH is inconsistent and limited to small observational studies with reported accuracy rates varying from $65-88 \%^{(26-28)}$. The evidence for the role of magnetic resonance imaging as a diagnostic tool for $A E H$ and concurrent $E A C$ is limited and requires further validation ${ }^{(29)}$. Our observation that severe AEH is an independent predictor of concurrent EAC supports the findings of a previous study ${ }^{(7)}$ and suggests that grading of AEH may be useful in identifying patients at higher risk of malignancy.

Importantly, only $34 \%$ of patients with AEH in our study had a concurrent EAC. If a systematic pelvic and para aortic lymphadenectomy were performed as part of surgical staging, this would have resulted in overtreatment, and potentially increased morbidity, for a majority of patients. Furthermore $94 \%$ of the patients with concurrent EAC had presumed stage $1 \mathrm{~A}$ or $1 \mathrm{~B}$ disease. The low rates of high-risk EAC in our study are similar to previously published literature $(19,30)$. However it is important to note that the morbidity of lymph node assessment is much lower with 
the introduction of sentinel node biopsy ${ }^{(31)}$. Given the relatively high risk of concurrent $E A C$ in women with severe $A E H$, there may be a role for performing sentinel lymph node biopsies in these patients.

Our study has several limitations that should be acknowledged. The study was conducted at a single institution and therefore the findings may not be generalizable. Additionally, its retrospective design has an inherent risk of selection bias. While this study was limited to a small sample size which may have resulted in type II statistical errors because of lack of power, effect sizes reported small effects for results which did not reach statistical significance. A further limitation is that AEH and low-grade endometrioid carcinoma are diagnoses that lie on a histopathological spectrum and the underdiagnosis of carcinoma on biopsy is possible. This may account for the high proportion of concurrent carcinomas in patients with severe $\mathrm{AEH}$ in pre-hysterectomy endometrial biopsies. The grading of $\mathrm{AEH}$ used in our practice is subjective and has not been validated externally and this is an important area for future research. Translational research that investigates molecular alterations according to AEH severity might provide insight into the risk of progression to malignancy in addition to histological grade.

Strengths of our study include its centralized state-wide tumour board that facilitated accurate case ascertainment, and central pathology review. To our knowledge this is the first study to assess the association of grade of AEH and its interaction with additional recognised risk factors for EAC.

In conclusion, in this study severe AEH and postmenopausal status were significant predictors of concurrent EAC in patients with $A E H$. If validated in larger prospective studies, gynaecologic oncologists may consider incorporating the grade of AEH to triage referrals within an appropriate timeframe and to perform sentinel lymph node biopsies for patients at high risk of concurrent EAC. However, we acknowledge that the recommendation for gynaecologic oncologists to perform surgery for all patients with severe AEH will add to an already overburdened system. 

Table 1. General Patient information.

\begin{tabular}{|c|c|c|c|c|}
\hline Variable & $\mathbf{n}$ & Mean & Median & SD \\
\hline Age at diagnosis (years) & 97 & 57.08 & 58.00 & 11.45 \\
\hline Weight (kg) & 97 & 97.51 & 100.00 & 24.12 \\
\hline Height (m) & 97 & 1.62 & 1.63 & 0.07 \\
\hline BMI & 97 & 36.98 & 37.50 & 8.23 \\
\hline Endometrial thickness (USS) a & 77 & 13.30 & 11.00 & 8.28 \\
\hline Days from diagnosis to treatment ${ }^{a}$ & 97 & 162.26 & 118.00 & 135.99 \\
\hline Carcinoma size $(\mathrm{mm})^{a}$ & 33 & 17.57 & 10.00 & 17.03 \\
\hline
\end{tabular}

${ }^{a}$ Not normally distributed as per Shapiro Wilk test 
Table 2. General Medical / Gynaecological History and Treatment undertaken on Patients ( $n=97)$

\begin{tabular}{|c|c|c|c|c|c|c|}
\hline \multirow[b]{2}{*}{ Variable } & \multirow[b]{2}{*}{ Frequency } & \multirow[b]{2}{*}{ Percent } & \multirow[b]{2}{*}{$\begin{array}{r}\text { Excluding } \\
\text { Missing }\end{array}$} & \multicolumn{3}{|c|}{$\begin{array}{c}\text { Carcinoma Group } \\
\text { Difference }^{\mathrm{a}}\end{array}$} \\
\hline & & & & $x^{2}$ & $\begin{array}{c}p- \\
\text { value }\end{array}$ & Effect Size $^{b}$ \\
\hline Diabetes & & & & 0.01 & 1.000 & 0.01 \\
\hline No & 64 & 66.0 & & & & \\
\hline Yes & 33 & 34.0 & & & & \\
\hline Hypertension & & & & 4.03 & .052 & 0.20 \\
\hline No & 40 & 41.2 & & & & \\
\hline Yes & 57 & 58.8 & & & & \\
\hline PCOS & & & & 2.20 & .256 & 0.17 \\
\hline No & 64 & 66.0 & 87.7 & & & \\
\hline Yes & 9 & 9.3 & 12.3 & & & \\
\hline Missing & 24 & 24.7 & & & & \\
\hline Smoking & & & & 0.65 & .805 & 0.08 \\
\hline Non-smoker & 64 & 66.0 & 66.7 & & & \\
\hline Smoker & 19 & 19.6 & 19.8 & & & \\
\hline Ex-smoker & 13 & 13.4 & 13.5 & & & \\
\hline Missing & 1 & 1.0 & & & & \\
\hline History of Cancer & & & & 2.97 & .768 & 0.18 \\
\hline None & 88 & 90.7 & & & & \\
\hline Breast & 6 & 6.2 & & & & \\
\hline Colorectal & 1 & 1.0 & & & & \\
\hline Urinary tract & 1 & 1.0 & & & & \\
\hline Other & 1 & 1.0 & & & & \\
\hline BMI groups & & & & 1.65 & .823 & 0.13 \\
\hline$<25$ & 11 & 11.3 & & & & \\
\hline $26-30$ & 11 & 11.3 & & & & \\
\hline $31-35$ & 18 & 18.6 & & & & \\
\hline $36-40$ & 24 & 24.7 & & & & \\
\hline$>40$ & 33 & 34.0 & & & & \\
\hline Parity & & & & 10.24 & .111 & 0.33 \\
\hline 0 & 15 & 15.5 & & & & \\
\hline 1 & 21 & 21.6 & & & & \\
\hline 2 & 36 & 37.1 & & & & \\
\hline 3 & 13 & 13.4 & & & & \\
\hline 4 & 6 & 6.2 & & & & \\
\hline 5 & 5 & 5.2 & & & & \\
\hline 7 & 1 & 1.0 & & & & \\
\hline Menopausal status & & & & 7.57 & *.017 & 0.28 \\
\hline Pre & 23 & 23.7 & & & & \\
\hline
\end{tabular}




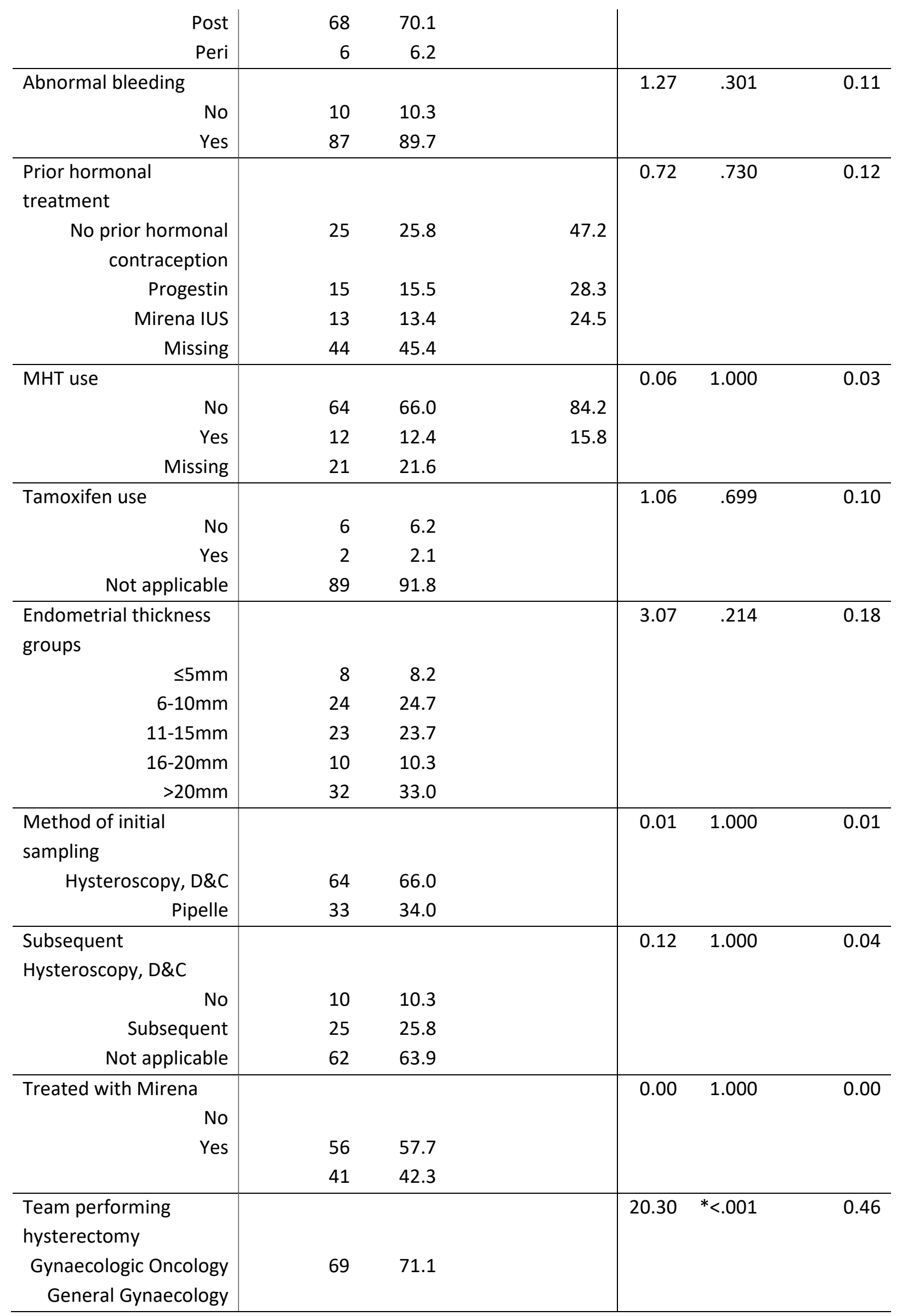




\begin{tabular}{|c|c|c|c|c|c|c|}
\hline & 28 & 28.9 & & & & \\
\hline Atypia & & & & 24.61 & $* .001$ & 0.52 \\
\hline Mild & 28 & 28.9 & 31.1 & & & \\
\hline Moderate & 29 & 29.9 & 32.2 & & & \\
\hline Severe & 33 & 34.0 & 36.7 & & & \\
\hline Missing & 7 & 7.2 & & & & \\
\hline $\begin{array}{l}\text { Suspicious for } \\
\text { malignancy }\end{array}$ & & & & 12.31 & $* .001$ & 0.36 \\
\hline Not suspicious & 84 & 86.8 & & & & \\
\hline Suspicious & 13 & 13.4 & & & & \\
\hline $\begin{array}{l}\text { Atypia only within } \\
\text { endometrial polyp }\end{array}$ & & & & 2.03 & .210 & 0.15 \\
\hline Not confined & 74 & 76.3 & & & & \\
\hline Confined & 23 & 23.7 & & & & \\
\hline
\end{tabular}

${ }^{a}$ Fisher's Exact Chi Square test with 2-sided p-value reported.

${ }^{b}$ Cramer's $V$ effect size reported

* statistically significant $\mathrm{p}<.05$

$\mathrm{MHT}=$ menopausal hormone therapy 
Table 3. Clinicopathological characteristics in patients with carcinoma $(n=33)$

\begin{tabular}{|c|c|c|}
\hline Variable & Frequency & Percent \\
\hline \multicolumn{3}{|l|}{ Carcinoma } \\
\hline Absent & 64 & 66.0 \\
\hline Present & 33 & 34.0 \\
\hline \multicolumn{3}{|l|}{ Final carcinoma histotype } \\
\hline Endometrioid & 31 & 93.9 \\
\hline Serous & 1 & 3.0 \\
\hline Mixed & 1 & 3.0 \\
\hline \multicolumn{3}{|l|}{ Carcinoma grade } \\
\hline 1 & 32 & 97.0 \\
\hline 3 & 1 & 3.0 \\
\hline \multicolumn{3}{|l|}{ Myoinvasion } \\
\hline No & 19 & 57.6 \\
\hline Inner half & 9 & 27.3 \\
\hline Outer half & 5 & 15.2 \\
\hline \multicolumn{3}{|l|}{ Carcinoma stage } \\
\hline $1 \mathrm{~A}$ & 27 & 81.8 \\
\hline $1 B$ & 4 & 12.1 \\
\hline $3 \mathrm{~A}$ & 1 & 3.0 \\
\hline $3 \mathrm{C} 1$ & 1 & 3.0 \\
\hline \multicolumn{3}{|l|}{ Lymphovascular space invasion } \\
\hline None & 31 & 93.9 \\
\hline Focal & 1 & 3.0 \\
\hline Extensive & 1 & 3.0 \\
\hline \multicolumn{3}{|l|}{ Lymph node status } \\
\hline Not assessed & 31 & 93.9 \\
\hline Negative & 1 & 3.0 \\
\hline Positive & 1 & 3.0 \\
\hline
\end{tabular}


Table 4. Final Binary Logistic Generalised Linear Model results

\begin{tabular}{|c|c|c|c|c|c|c|}
\hline \multirow[t]{2}{*}{ Parameter } & \multirow[t]{2}{*}{$\begin{array}{r}\beta \\
\text { estimate }\end{array}$} & \multirow[t]{2}{*}{ SE } & \multirow[t]{2}{*}{$\operatorname{Exp}(\beta)$} & \multicolumn{2}{|c|}{$\begin{array}{c}95 \% \text { Wald } \\
\text { Confidence Interval } \\
\operatorname{Exp}(\beta)\end{array}$} & \multirow[b]{2}{*}{$\begin{array}{r}p- \\
\text { value }\end{array}$} \\
\hline & & & & Lower & Upper & \\
\hline Intercept & -1.78 & 2.94 & 0.2 & 0.0 & 53.6 & .546 \\
\hline \multicolumn{7}{|l|}{ Atypia } \\
\hline Severe $^{1}$ & 3.07 & 1.03 & 21.5 & 2.8 & 163.1 & $* .003$ \\
\hline Moderate ${ }^{1}$ & 0.72 & 0.94 & 2.0 & 0.3 & 13.0 & .448 \\
\hline Suspicious for malignancy ${ }^{2}$ & 1.09 & 1.41 & 3.0 & 0.2 & 47.3 & .438 \\
\hline Hypertension ${ }^{3}$ & 0.71 & 0.84 & 2.0 & 0.4 & 10.5 & .393 \\
\hline Postmenopausal status ${ }^{4}$ & 2.58 & 1.20 & 13.2 & 1.3 & 139.0 & $* .032$ \\
\hline Endometrial thickness & 0.05 & 0.04 & 1.1 & 1.0 & 1.1 & .259 \\
\hline Age at diagnosis & -0.03 & 0.04 & 1.0 & 0.9 & 1.1 & .475 \\
\hline BMI & -0.05 & 0.05 & 1.0 & 0.9 & 1.1 & .365 \\
\hline
\end{tabular}


Figure 1. Representative images of mild (A), moderate (B) and severe (C) atypical hyperplasia showing increasing gland to stromal ratio with increasing grade. Nonhyperplastic mucosa is present at the right of each image for comparison. (D) Cytological alteration in atypical hyperplasia (left) compared to normal endometrial gland (right). 


\section{Referrences}

1. Sanderson PA, Critchley HO, Williams AR, Arends MJ, Saunders PT. New concepts for an old problem: the diagnosis of endometrial hyperplasia. Hum Reprod Update. 2017;23(2):232-54.

2. WHO Classification of Tumours of Female Reproductive Organs [press release]. Lyon (France): International Agency for Research on Cancer2020.

3. Kurman RJ, Kaminski PF, Norris HJ. The behavior of endometrial hyperplasia. A long-term study of "untreated" hyperplasia in 170 patients. Cancer. 1985;56(2):403-12.

4. Terence J. Colgan CMM. Comprehensive Cytopathology. 3rd ed: Elsevier; 2008.

5. Trimble CL, Kauderer J, Zaino R, Silverberg S, Lim PC, Burke JJ, 2nd, et al. Concurrent endometrial carcinoma in women with a biopsy diagnosis of atypical endometrial hyperplasia: a Gynecologic Oncology Group study. Cancer. 2006;106(4):812-9.

6. Lacey JV, Jr., Sherman ME, Rush BB, Ronnett BM, Ioffe OB, Duggan MA, et al. Absolute risk of endometrial carcinoma during 20-year follow-up among women with endometrial hyperplasia. Journal of clinical oncology : official journal of the American Society of Clinical Oncology. 2010;28(5):788-92.

7. Rakha E, Wong SC, Soomro I, Chaudry Z, Sharma A, Deen S, et al. Clinical outcome of atypical endometrial hyperplasia diagnosed on an endometrial biopsy: institutional experience and review of literature. The American journal of surgical pathology. 2012;36(11):1683-90.

8. Matsuo K, Ramzan AA, Gualtieri MR, Mhawech-Fauceglia P, Machida H, Moeini A, et al. Prediction of concurrent endometrial carcinoma in women with endometrial hyperplasia. Gynecologic oncology. 2015;139(2):261-7.

9. Yin J, Ma S, Shan Y, Wang Y, Li Y, Jin Y, et al. Risk Factors for Recurrence in Patients with Atypical Endometrial Hyperplasia and Endometrioid Adenocarcinoma after Fertility-Sparing Treatments. Cancer Prev Res (Phila). 2020;13(4):403-10.

10. Philip Clement JS, Robert Young. Atlas of Gynecologic Surgical Pathology. 4th ed: Elsevier; 2019 9th April 2019. 670 p.

11. Reed SD, Newton KM, Clinton WL, Epplein M, Garcia R, Allison K, et al. Incidence of endometrial hyperplasia. American journal of obstetrics and gynecology. 2009;200(6):678.e1-6.

12. Management of endometrial hyperplasia [Internet]. Green-to Guideline, Royal college of obstetricians and gynaecologist. 2016 [cited 20.04.2019]. Available

from:

https://www.rcog.org.uk/globalassets/documents/guidelines/green-topguidelines/gtg 67 endometrial hyperplasia.pdf.

13. Giannella L, Cerami LB, Setti T, Bergamini E, Boselli F. Prediction of Endometrial Hyperplasia and Cancer among Premenopausal Women with Abnormal Uterine Bleeding. BioMed research international. 2019;2019:8598152. 14. Rungruang $\mathrm{B}$, Olawaiye $\mathrm{AB}$. Comprehensive surgical staging for endometrial cancer. Rev Obstet Gynecol. 2012;5(1):28-34.

15. Mariani A, Dowdy SC, Cliby WA, Gostout BS, Jones MB, Wilson TO, et al. Prospective assessment of lymphatic dissemination in endometrial cancer: a paradigm shift in surgical staging. Gynecologic oncology. 2008;109(1):11-8. 
16. Rossi EC, Kowalski LD, Scalici J, Cantrell L, Schuler K, Hanna RK, et al. A comparison of sentinel lymph node biopsy to lymphadenectomy for endometrial cancer staging (FIRES trial): a multicentre, prospective, cohort study. Lancet Oncol. 2017;18(3):384-92.

17. Diaz-Montes TP, Zahurak ML, Giuntoli RL, 2nd, Gardner GJ, Bristow RE. Uterine cancer in Maryland: impact of surgeon case volume and other prognostic factors on short-term mortality. Gynecologic oncology. 2006;103(3):1043-7.

18. Antonsen SL, Ulrich L, Hogdall C. Patients with atypical hyperplasia of the endometrium should be treated in oncological centers. Gynecologic oncology. 2012;125(1):124-8.

19. Gungorduk K, Ozdemir A, Ertas IE, Sahbaz A, Asicioglu O, Gokcu M, et al. A novel preoperative scoring system for predicting endometrial cancer in patients with complex atypical endometrial hyperplasia and accuracy of frozen section pathological examination in this context: a multicenter study. Gynecologic and obstetric investigation. 2015;79(1):50-6.

20. Zhang C, Wang EY, Liu F, Sung CJ, Quddus MR, Ou J, et al. Routine histologic features in complex atypical hyperplasia can predict the presence of endometrial carcinoma: a clinicopathological study of 222 cases. Human pathology. 2018;80:40-6.

21. Melon J, Leung Y, Salfinger SG, Tan J, Mohan G, Cohen PA. Value of Specialist Pathology Review in a Single Statewide Gynecologic Cancer Service. Int J Gynecol Cancer. 2017;27(1):171-6.

22. Stewart CJR, Leung YC, Chaudry Z, Koay MHE, Naran A, Plunkett M, et al. Evaluation of pathology review at gynaecological oncology multidisciplinary team meetings: a 5-year prospective analysis of cases with major diagnostic discordance. Pathology. 2019;51(4):353-61.

23. Calculation of Effect Sizes [Internet]. Psychometrica. 2016. Available from: https://www.psychometrica.de/effect size.html. .

24. Touboul C, Piel B, Koskas M, Gonthier C, Ballester M, Cortez A, et al. Factors predictive of endometrial carcinoma in patients with atypical endometrial hyperplasia on preoperative histology. Anticancer research. 2014;34(10):5671-6.

25. Leitao MM, Jr., Kehoe S, Barakat RR, Alektiar K, Gattoc LP, Rabbitt C, et al. Comparison of D\&C and office endometrial biopsy accuracy in patients with FIGO grade 1 endometrial adenocarcinoma. Gynecologic oncology. 2009;113(1):105-8. 26. Indermaur MD, Shoup B, Tebes S, Lancaster JM. The accuracy of frozen pathology at time of hysterectomy in patients with complex atypical hyperplasia on preoperative biopsy. American journal of obstetrics and gynecology. 2007;196(5):e40-2.

27. Morotti M, Menada MV, Moioli M, Sala P, Maffeo I, Abete L, et al. Frozen section pathology at time of hysterectomy accurately predicts endometrial cancer in patients with preoperative diagnosis of atypical endometrial hyperplasia. Gynecologic oncology. 2012;125(3):536-40.

28. Attard Montalto S, Coutts M, Devaja O, Summers J, Jyothirmayi R, Papadopoulos A. Accuracy of frozen section diagnosis at surgery in premalignant and malignant lesions of the endometrium. European journal of gynaecological oncology. 2008;29(5):435-40. 
29. Natarajan P, Vinturache A, Hutson R, Nugent D, Broadhead T. The value of MRI in management of endometrial hyperplasia with atypia. World J Surg Oncol. 2020;18(1):34.

30. Costales AB, Schmeler KM, Broaddus R, Soliman PT, Westin SN, Ramirez $\mathrm{PT}$, et al. Clinically significant endometrial cancer risk following a diagnosis of complex atypical hyperplasia. Gynecologic oncology. 2014;135(3):451-4.

31. Holloway RW, Abu-Rustum NR, Backes FJ, Boggess JF, Gotlieb WH, Jeffrey Lowery W, et al. Sentinel lymph node mapping and staging in endometrial cancer: A Society of Gynecologic Oncology literature review with consensus recommendations. Gynecologic oncology. 2017;146(2):405-15. 